\title{
Uncountability of Real Numbers
}

\author{
Reema Agarwal ${ }^{1}$, Mahesh Kumar ${ }^{2}$ \\ 1(Department of Mathematics, Lady Shri Ram College For Women, University of Delhi, India) \\ 2(Department of Mathematics, Lady Shri Ram College For Women, University of Delhi, India)
}

\begin{abstract}
In this article, it will be shown that the set of Real numbers is uncountable in four different ways. The first one uses the least upper bound property of the set of real numbers $\mathbb{R}$ (sometimes called the completeness property of $\mathbb{R})$, the second one uses the nested intervals property of $\mathbb{R}$, the third one uses Cantor's diagonal argument and the fourth one by proving that a non-empty perfect subset of $\mathbb{R}$ is uncountable.
\end{abstract}

Keywords:Uncountable set, monotone sequences, bounded above, bounded below, perfect set, neighbourhood of a point, limit point of a set.

\section{Introduction}

In this article the Uncountabilityof $\mathbb{R}$, the set of real numbers is shown in four different ways and each time one can observe that the completeness property of $\mathbb{R}$ is very much needed to prove that $\mathbb{R}$ is uncountable. In the first proof the completeness property of $\mathbb{R}$ sometimes called the least upper bound property of $\mathbb{R}$ plays a crucial role in order to show that $\mathbb{R}$ is uncountable. Similarly the second proof uses the nested intervals property of $\mathbb{R}$ which is just another version of the completeness property of $\mathbb{R}$ and the third one uses the so- called Cantor's diagonal argument.Here it is pointed out that in this proof the fact that for each $x \in[0,1]$ there is a sequence of integers $\left(a_{n}\right)$ with $0 \leq a_{n} \leq 9$ for all $n$ such that $x=0 . a_{1} a_{2} \ldots . a_{n} \ldots .$. has been used, the proof of which again uses the least upper bound property of $\mathbb{R}[$ see 3$]$.

Finally the fourth proof uses that a non- empty perfect subset of $\mathbb{R}$ is uncountable whose proof again uses another version of the completeness property of $\mathbb{R}$ (in the sense of Cantor), viz., A sequence of real numbers converges if and only if it is Cauchy.

The article is self contained and any prerequisites needed for thefour proofs have been given in the section below.

The following is recalled

\section{Some Basic Definitions and Results}

Definition 1: A set $\mathcal{A}$ is called a finite set if $\mathcal{A}=\phi$ or if it is in one to one correspondence with the $\operatorname{set}\{1,2,3, \ldots \ldots, n\}$ for some $\in \mathbb{N}$; otherwise we say that $\mathcal{A}$ is infinite.

Definition 2: An infinite set $\mathcal{A}$ is said to be countable or countably infinite if $\mathcal{A}$ is in one to one correspondence with the set of Natural numbers $\mathbb{N}$. That is, the elements of a countable set $\mathcal{A}$ can be enumerated or counted according to their correspondence with the natural numbers: $\mathcal{A}=\left\{x_{1}, x_{2}, x_{3}, \ldots.\right\}$ where the $x_{i}^{\prime} s$ are distinct.

Definition 3: An infinite set that is not countable is called uncountable.

Definition 4: A subset $\mathcal{A}$ of $\mathbb{R}$ is said to be bounded above if there is some $x \in \mathbb{R}$ such that $a \leq x$. for all $a \in \mathcal{A}$. Any such number $x$ is called an upper bound for $\mathcal{A}$.

Theorem: (The Least Upper Bound Property of $\mathbb{R}$ (Sometimes called the Completeness Property of $\mathbb{R}$ )). Any nonempty set of real numbers with an upper bound has a least upper bound.

That is, if $A \subseteq \mathbb{R}$ is nonempty and bounded above, then there is a number $s \in \mathbb{R}$ satisfying: (i) $s$ is an upper bound for $A$; and (ii) if $x$ is any upper bound for $A$, then $s \leq x$. In this case we write $s=l . u . b . A=\sup A$ (for supremum). Similarly, we also have greatest lower bounds (g.l.b.) of a set $A \subseteq \mathbb{R}$, denoted by $\inf A$ (for infimum).

Definition 5: A set $A \subseteq \mathbb{R}$ which is both bounded above and bounded below is called bounded.

Theorem: (Nested Intervals Property). If $I_{n}=\left[a_{n}, b_{n}\right], n \in \mathbb{N}$ is a nested sequence of closed bounded intervals, i.e $I_{1} \supseteq I_{2} \supseteq \ldots \ldots . \supseteq I_{n} \ldots \ldots$, then there exists a number $\xi \in \mathbb{R}$ such that $\xi \in I_{n}$ for all $n \in \mathbb{N}$.

Definition 6: A sequence $\left(x_{n}\right)$ of real numbers is said to converge to $x \in \mathbb{R}$ if, for every $\varepsilon>0$, there is a positive integer $N$ such that $\left|x_{n}-x\right|<\varepsilon$ whenever $n \geq N$. In this case, we call $x$ the limit of the sequence $\left(x_{n}\right)$ and write $x=\lim _{n \rightarrow \infty} x_{n}$. 
Theorem: (Monotone Convergence Theorem).A monotone sequence of real numbers is convergent if and only if it is bounded. Further, If $\left(x_{n}\right)$ is a bounded increasing sequence, then $\lim _{n \rightarrow \infty} x_{n}=\sup \left\{x_{n}: n \in \mathbb{N}\right\}$ and if $\left(y_{n}\right)$ is a bounded decreasing sequence, thenlim $\lim _{n \rightarrow \infty} y_{n}=\inf \left\{y_{n}: n \in \mathbb{N}\right\}$.

Definition 7: A sequence $\left(x_{n}\right)$ of real numbers is said to be Cauchy if, for every $\varepsilon>0$, there is a positive integer $N$ such that $\left|x_{n}-x_{m}\right|<\varepsilon$ whenever $n, m \geq N$.

Theorem: (Completeness Property of $\mathbb{R}$ in the sense of Cantor).A sequence of real numbers converges if and only if it is Cauchy.

Definition 8: A neighbourhood of a point $x$ is a set $N_{r}(x)$ consisting of all $y$ such that $|x-y|<r$.

Definition 9: A point $x$ is a limit point of the set $E \subseteq \mathbb{R}$ if every neighbourhood of $x$ contains a point $y \neq x$ such that $y \in E$.

Definition 10: Let $E \subseteq \mathbb{R}$. Then $E$ is called a perfect set if $E$ is closed and if every point of $E$ is a limit point of E.

\section{The set $\mathbb{R}$ of real numbers is uncountable.}

Now the four proofs of the main result are given. In the first proof below the completeness property of $\mathbb{R}$ (sometimes called the least upper bound property of $\mathbb{R}$ ) plays a crucial role in the form of Monotone Convergence Theorem in order to show that $\mathbb{R}$ is uncountable.

Proof 1: It is enough to prove that the set $[0,1]$ is uncountable because then $\mathbb{R}$ being a superset of $[0,1]$ will definitelybe uncountable as a superset of an uncountable set is uncountable. If possible, let us assume that $[0,1]$ is countable. Since $[0,1]$ is infinite (as $\frac{1}{n} \in[0,1] \forall n \in \mathbb{N}$ ), there exists a bijection (one to one correspondence) $f: \mathbb{N} \rightarrow[0,1]$. Let $z_{n}=f(n)$. We prove that there exists $x \in[0,1]$ such that $x \neq z_{n}$ for any $n \in \mathbb{N}$ and we are done then.

Now two sequences $\left(x_{n}\right)$ and $\left(y_{n}\right)$ are defined whoses elements are defined recursively.

Let $x_{1}$ be the $z_{r}$ where $r$ is the first integer such that $0<z_{r}<1$. Let $y_{1}$ be $z_{s}$ where $s$ is the first integer such that $x_{1}<z_{s}<1$.

Assume that $\left(x_{i}\right)_{i=1}^{n}$ and $\left(y_{i}\right)_{i=1}^{n}$ have been chosen with the property that

$$
0=x_{0}<x_{1}<x_{2}<\ldots \ldots \ldots x_{n}<y_{n}<y_{n-1}<\ldots \ldots<y_{2}<y_{1}<y_{0}=1
$$

Now choose $x_{n+1}$ to be the $z_{r}$ where $r$ is the first integer such that $x_{n}<z_{r}<y_{n}$. Let $y_{n+1}$ be $z_{s}$ where $s$ is the first integer such that $x_{n+1}<z_{s}<y_{n}$. Note that if no such $r$ ors exists at some stage then actually we are done as then an element $x$ can easily be chosen in $\left[x_{n}, y_{n}\right]$ such that $x \neq z_{n} \forall n \in \mathbb{N}$.

Thus we have sequences $\left(x_{n}\right)_{n \in \mathbb{N}}$ and $\left(y_{n}\right)_{n \in \mathbb{N}}$ with $x_{1}<x_{2}<\cdots<y_{2}<y_{1}$. So $\left(x_{n}\right)_{n \in \mathbb{N}}$ and $\left(y_{n}\right)_{n \in \mathbb{N}}$ are monotone and bounded above and below respectively. Therefore by the monotone convergence theorem both are convergent.

Let $x=\lim _{n \rightarrow \infty} x_{n}=\sup \left\{x_{n}\right\}$ and $y=\lim _{n \rightarrow \infty} y_{n}=\inf \left\{y_{n}\right\}$. Then as $x_{n}<y_{n}$ for each $n \in \mathbb{N}$, we have $x \leq y$, as $[x, y] \neq \phi$.

Leth $\in[x, y]$. Then $h \neq x_{n}, y_{n} \forall n \in \mathbb{N}$, since $x_{n}<h<y_{n}$ for all $n \in \mathbb{N}$. We now claim that $h \neq z_{n}$ for all $n \in \mathbb{N}$. Suppose that $h=z_{n}$ for some $n \in \mathbb{N}$. Then there are only finitely many points in the sequence $\left(z_{n}\right)_{n \in \mathbb{N}}$ before $h$ occurs, and therefore only finitely of the sequence $\left(x_{n}\right)_{n \in \mathbb{N}}$ precedes $h$.

Let $x_{d}$ be the last element of the sequence $\left(x_{n}\right)_{n \in \mathbb{N}}$ preceding $h$. Then by definition $x_{d+1}, y_{d+1}$ are interior points of $\left[x_{d}, y_{d}\right]$ and also $h \in\left[x_{d+1}, y_{d+1}\right]$ by the definition of $h$.

Therefore $x_{d+1}$ must precede $h$ in the sequence, for the sequence is monotonically increasing, a contradiction since $x_{d}$ was the last element of the sequence $\left(x_{n}\right)_{n \in \mathbb{N}}$ preceding $h$. Therefore our assumption that $h=z_{n}$ for some $n \in \mathbb{N}$ is wrong. Hence $h \neq z_{n}$ for any $n \in \mathbb{N}$, and thus $[0,1]$ is not countable and hence uncountable.

In the second proof, the Nested Interval Property is used which was given by Georg Cantor in 1874 in the first of his papers on infinite sets. He later published a proof that used decimal representation of real numbers and that proof is given after this proof.

Proof : Again it will be proved that $I=[0,1]$ is an uncountable set.

Let, if possible $I$ be countable. Then we can enumerate the set as $I=\left\{x_{1}, x_{2}, \ldots \ldots \ldots, x_{n}, \ldots \ldots.\right\}$ where all $x_{i}{ }^{\prime} s$ are distinct.

First a closed subinterval $I_{1}$ of $I$ is selected such that $x_{1} \notin I_{1}$. Note that $I_{1}$ can be selected easily by dividing $I$ into three equal parts. Then, similarly, a closed subinterval $I_{2}$ of $I_{1}$ is selected such that $x_{2} \notin I_{2}$ and so on.

In this way non empty closed intervals are obtained 
such that $I_{n} \subseteq I$ and $x_{n} \notin I_{n}$ for all $n \in \mathbb{N}$.

$$
I_{1} \supseteq I_{2} \supseteq I_{3} \supseteq \cdots \ldots I_{n} \ldots
$$

The nested intervals property implies that there exists a point $\xi \in I$ such that $\xi \in I_{n}$ for all $n \in \mathbb{N}$. Therfore, $\xi \neq x_{n}$ for all $n \in \mathbb{N}$. So the enumeration of $\mathrm{I}$ is not a complete listing of the elements of $I$, as claimed.Hence, $I$ is an uncountable set.

Now we give Cantor's another proof of the uncountability of $\mathbb{R}$ which was mentioned above.This is an elegant "diagonal" argument based on decimal representation of real numbers.

Proof 3:It will be proved that $[0,1]$ is not countable. Again the proof is by contradiction. The fact that every real number $x \in[0,1]$ has a decimal representation $x=0 . b_{1} b_{2} b_{3} \ldots \ldots \ldots \ldots$. where $b_{i}=0,1,2,3 \ldots . .9$ will be used. It is pointed out that this fact is actually a consequence of the least upper bound property of $\mathbb{R}$ [see 3$]$.

Suppose that there is an enumeration $x_{1}, x_{2}, x_{3}, \ldots \ldots \ldots$ of all numbers in $[0,1]$ which can be displaced as:

$$
\begin{aligned}
& x_{1}=0 . b_{11} b_{12} b_{13} \ldots \ldots . b_{1 n} \ldots \text {, } \\
& x_{2}=0 . b_{21} b_{22} b_{23} \ldots \ldots . b_{2 n} \ldots \text {, } \\
& x_{3}=0 . b_{31} b_{32} b_{33} \ldots \ldots \ldots b_{3 n \ldots .} \text {, } \\
& \text {..... } \quad \ldots \ldots \ldots \\
& x_{n}=0 . b_{n 1} b_{n 2} b_{n 3} \ldots \ldots \ldots b_{n n} \cdots
\end{aligned}
$$

Now a real number $y=0 . y_{1} y_{2} \ldots \ldots y_{n} \ldots$ is defined by setting

$$
y_{n}= \begin{cases}1, & \text { if } b_{n n} \geq 5 \\ 7, & \text { if } b_{n n} \leq 4\end{cases}
$$

Note that here 1 and 7 have been chosen more or less arbitrarily just to avoid the troublesome digits 0 and 9 so that the decimal representation of $y$ is then unique because it does not end in all $0^{\prime} s$ or all $9^{\prime} s$, since $y_{n} \neq$ 0,9 for all $n \in \mathbb{N}$.

Further, since $y$ and $x_{n}$ differs in the $n^{\text {th }}$ decimal place, $y \neq x_{n}$ for any $n \in \mathbb{N}$. Therefore, $y$ is not included in the enumeration of $[0,1]$, contradicting the hypothesis.

Proof 4: It is enough to prove that if $P$ is any non empty perfect set in $\mathbb{R}$, then $P$ is uncountable, because then $[0,1]$ will also be uncountable as $[0,1]$ is a perfect set.

So, let $P$ be a nonempty perfect set in $\mathbb{R}$. Since $P$ has limit points, $P$ must be infinite (since a finite set has no limit point).

Let, if possible, $P$ be countable. Denote the points of $P$ by $x_{1}, x_{2}, x_{3}, \ldots$. . Let $y_{1} \in P$ and Let $N_{1}:=\overline{\left(N_{r}\left(y_{1}\right)\right)}$ be some closed neighbourhood centered at $y_{1}$ such that $x_{1} \notin N_{1}$. Given $N_{n-1}$ choose a closed neighbourhood $N_{n-1} \supseteq N_{n}:=N_{\delta(n)}\left(y_{n}\right)$, such that $\delta(n) \leq \frac{\delta(n-1)}{2} \quad$ (note that $\left.\delta(1)=r\right), y_{n} \in X$ and $x_{n} \notin$ $N_{n}$. The condition $x_{n} \notin N_{n}$ can be satisfied because $P$ is perfect, so every neighbourhood centered at a point of $P$ contains infinitely many points of $P$. Since $\delta(n) \leq \frac{r}{2^{n-1}},\left\langle y_{n}>\right.$ is Cauchy and hence convergent in $P$, since a perfect set is necessarilyclosed.

So, Let $y=\lim _{n \rightarrow \infty} y_{n} \in P$. For $n \in \mathbb{N}$, we have $\left\{y_{m}: m>n\right\} \subseteq N_{n}$. So $y \in N_{n}$ for each $n \in \mathbb{N}$. But by construction, $x_{n} \notin N_{n}$ for each $\in \mathbb{N}$. Therefore $y \neq x_{n}$ for all $n \in \mathbb{N}$, and so we are done.

\section{Conclusion}

In establishing the Uncountability of $\mathbb{R}$, the set of real numbers, even in four different ways, each time one would have observed that the completeness property of $\mathbb{R}$ is very much needed to prove that $\mathbb{R}$ is uncountable which is the most subtle point of any proof required for proving that $\mathbb{R}$ is uncountable.

\section{Books:}

\section{References}

[1]. W.Rudin,PrinciplesofMathematical Analysis, Third Edition, 1976.

[2]. C.G. Denlinger, Elements of Real Analysis, First Indian Edition, 2011.

[3]. N.L.Carothers, RealAnalysis, First South Asian Edition, 2006

[4]. Bartle \&Sherbert, Introduction to Real Analysis, Third Edition

[5]. G.F. Simmons, Introduction to Topology and Modern Analysis, Sixteenth reprint, 2011.

[6]. S.Kumaresan, Expository Articles, Notes and Exercise Sets. 